PROCEEDINGS OF THE

AMERICAN MATHEMATICAL SOCIETY

Volume 126, Number 11, November 1998, Pages 3377-3380

S $0002-9939(98) 04653-X$

\title{
AN EASIER PROOF OF THE MAXIMAL ARCS CONJECTURE
}

\author{
SIMEON BALL AND AART BLOKHUIS \\ (Communicated by Jeffry N. Kahn)
}

\begin{abstract}
It was a long-standing conjecture in finite geometry that a Desarguesian plane of odd order contains no maximal arcs. A rather inaccessible and long proof was given recently by the authors in collaboration with Mazzocca. In this paper a new observation leads to a greatly simplified proof of the conjecture.
\end{abstract}

\section{INTRODUCTION}

$\mathrm{A}(k, n)-$ arc in a projective plane is a set of $k$ points, at most $n$ on every line. If the order of the plane is $q$, then $k \leq 1+(q+1)(n-1)=q n-q+n$, with equality if and only if every line intersects the arc in 0 or $n$ points. Arcs realizing the upper bound are called maximal arcs. Equality in the bound implies that $n \mid q$ or $n=q+1$. If $1<n<q$, then the maximal arc is called non-trivial. The only known examples of non-trivial maximal arcs in Desarguesian projective planes are the hyperovals $(n=2)$, and, for $n>2$, the Denniston arcs [3] and an infinite family constructed by Thas [5], [7]. These exist for all pairs $(n, q)=\left(2^{a}, 2^{b}\right), 0<a<b$. It is conjectured in [6] that for odd $q$ maximal arcs do not exist. In that paper this was proved for $(n, q)=\left(3,3^{h}\right)$. The special case $(n, q)=(3,9)$ was settled earlier by Cossu [2]. A complete proof was given in [1], however the methods used there are difficult to follow and the arguments are quite long.

A new observation, concerning a divisibility relation between a function $F$ and its partial derivative $F_{x}$, led to the discovery of a greatly simplified proof which should be accessible to a wider audience.

We shall consider point sets in the affine plane $A G(2, q)$ instead of $P G(2, q)$. This is no restriction; there is always a line disjoint from a non-trivial maximal arc. The points of $A G(2, q)$ can be identified with the elements of $G F\left(q^{2}\right)$ in a suitable way, so that in fact all point sets can be considered as subsets of this field. Note that three points $a, b, c$ are collinear precisely when $(a-b)^{q-1}=(a-c)^{q-1}$. If the direction of the line joining $a$ and $b$ is identified with the number $(a-b)^{q-1}$, then a one-to-one correspondence between the $q+1$ directions (or parallel classes) and the different $(q+1)$-st roots of unity in $G F\left(q^{2}\right)$ is obtained.

\section{Some USEFul POLYNOMials}

Let $\mathcal{B}$ be a non-trivial $(n q-q+n, n)$-arc in $A G(2, q) \simeq G F\left(q^{2}\right), q=p^{h}$. For simplicity we assume $0 \notin \mathcal{B}$. Let $\mathcal{B}^{[-1]}=\{1 / b \mid b \in \mathcal{B}\}$. Define $B(x)$ to be the

Received by the editors March 23, 1997.

1991 Mathematics Subject Classification. Primary 51E21; Secondary 05B25. 
polynomial

$$
B(x):=\prod_{b \in \mathcal{B}}(1-b x)=\sum_{k=0}^{\infty}(-1)^{k} \sigma_{k} x^{k},
$$

where $\sigma_{k}$ denotes the $k$-th elementary symmetric function of the set $\mathcal{B}$; in particular, $\sigma_{k}=0$ for $k>|\mathcal{B}|$. Define the polynomials $F$ in two variables and $\hat{\sigma}_{k}$ in one variable by

$$
F(t, x):=\prod_{b \in \mathcal{B}}\left(1-(1-b x)^{q-1} t\right)=\sum_{k=0}^{\infty}(-1)^{k} \hat{\sigma}_{k} t^{k},
$$

where $\hat{\sigma}_{k}$ is the $k$-th elementary symmetric function of the set of polynomials $\left\{(1-b x)^{q-1} \mid b \in \mathcal{B}\right\}$, a polynomial of degree at most $k(q-1)$ in $x$. Again, $\hat{\sigma}_{k}$ is the zero polynomial for $k>|\mathcal{B}|$. For $x_{0} \in G F\left(q^{2}\right) \backslash \mathcal{B}^{[-1]}$ it follows that $F\left(t, x_{0}\right)$ is an $n$-th power. Indeed, if $x_{0}=0$ this is clear, and if $x_{0} \neq 0$ then $1 / x_{0}$ is a point not contained in the arc, so that every line through $1 / x_{0}$ contains a number of points of $\mathcal{B}$ that is either 0 or $n$. In the multiset $\left\{\left(1 / x_{0}-b\right)^{q-1} \mid b \in \mathcal{B}\right\}$, every element occurs therefore with multiplicity $n$, so that in $F\left(t, x_{0}\right)$ every factor occurs exactly $n$ times. For $x_{0} \in \mathcal{B}^{[-1]}$ we get that $F\left(t, x_{0}\right)=\left(1-t^{q+1}\right)^{n-1}$, for in this case every line passing through the point $1 / x_{0}$ contains exactly $n-1$ other points of $\mathcal{B}$, so that the multiset $\left\{\left(1 / x_{0}-b\right)^{q-1}\right\}$ consists of every $(q+1)$-st root of unity repeated $n-1$ times, together with the element 0 . This gives

$$
F\left(t, x_{0}\right)=\prod_{b \in \mathcal{B}}\left(1-\left(1 / x_{0}-b\right)^{q-1} x_{0}^{q-1} t\right)=\left(1-x_{0}^{q^{2}-1} t^{q+1}\right)^{n-1}=\left(1-t^{q+1}\right)^{n-1} .
$$

From the shape of $F$ in both cases it can be seen that for all $x_{0} \in G F\left(q^{2}\right)$, $\hat{\sigma}_{k}\left(x_{0}\right)=0,0<k<n$, and since the degree of $\hat{\sigma}_{k}$ is at most $k(q-1)<q^{2}$, these functions are in fact identically zero. The first coefficient of $F$ that is not necessarily identically zero therefore is $\hat{\sigma}_{n}$. Since $\hat{\sigma}_{n}(0)=\left(\begin{array}{c}|\mathcal{B}| \\ n\end{array}\right)=\left(\begin{array}{c}n q-q+n \\ n\end{array}\right)=1$, by Lucas' theorem, it is not identically zero. On the other hand the coefficient of $t^{n}$ in $\left(1-t^{q+1}\right)^{n-1}$ is zero, so $\hat{\sigma}_{n}\left(x_{0}\right)=0$ for $x_{0} \in \mathcal{B}^{[-1]}$. In other words, $B$ divides $\hat{\sigma}_{n}$.

Let $z=x-x^{q^{2}}$. Since in both cases, i.e. for all $x_{0} \in G F\left(q^{2}\right), \hat{\sigma}_{k}$ vanishes unless $n \mid k$ or $(q+1) \mid k$, it follows that $z \mid \hat{\sigma}_{k}$. If $n \nmid k$, then $\hat{\sigma}_{k}$ still vanishes for $x_{0} \in G F\left(q^{2}\right) \backslash \mathcal{B}^{[-1]}$, and since $B \mid \hat{\sigma}_{n}$ we get the divisibility relation $\left(x-x^{q^{2}}\right) \mid \hat{\sigma}_{n} \hat{\sigma}_{k}$. Hence we can write

$$
F(t, x)=1+\sum_{i=1}^{q-q / n+1}(-1)^{i} \hat{\sigma}_{i n} t^{i n}+\sum_{i=1}^{n-1} \hat{\sigma}_{i(q+1)} t^{i(q+1)} \quad(\bmod z)
$$

and

$$
B F(t, x)=B+B \sum_{i=1}^{q-q / n+1}(-1)^{i} \hat{\sigma}_{i n} t^{i n} \quad(\bmod z) .
$$

The polynomial $\hat{\sigma}_{q+1}$ will be of some use as well, so it is worth noting that $\hat{\sigma}_{q+1}\left(x_{0}\right)=1$ for all $x_{0} \in \mathcal{B}^{[-1]}$ and $\hat{\sigma}_{q+1}\left(x_{0}\right)=0$ for all $x_{0} \in G F\left(q^{2}\right) \backslash \mathcal{B}^{[-1]}$.

\section{Proof of the theorem}

The main objective of the proof is to show that $\left(B \hat{\sigma}_{n}\right)^{\prime} \equiv 0$, which will lead swiftly to a contradiction for $p \neq 2$. Throughout, $f^{\prime}$ will represent the derivative of a function $f$ with respect to $x$, and $f_{x}$ will denote the partial derivative with respect to $x$. 
By computing the derivative of $B(x)$ and expanding the denominator as an infinite sum we get

$$
B^{\prime}(x)=\sum_{b \in \mathcal{B}} \frac{-b}{1-b x} B(x)=-\left(\sum_{b \in \mathcal{B}} \sum_{i=0}^{\infty} b^{i+1} x^{i}\right) B(x) .
$$

Note that all $b \in \mathcal{B}^{[-1]}$ are elements of $G F\left(q^{2}\right)$. Hence $b^{q^{2}}=b$, and it follows that

$$
\left(x-x^{q^{2}}\right)\left(\sum_{b \in \mathcal{B}} \sum_{i=0}^{\infty} b^{i+1} x^{i}\right)=\sum_{b \in \mathcal{B}} \sum_{i=0}^{q^{2}-1} b^{i} x^{i}=\sum_{b \in \mathcal{B}}(1-b x)^{q^{2}-1} .
$$

The polynomial $-\sum_{b \in \mathcal{B}}(1-b x)^{q^{2}-1}$ is equal to 1 for all $x_{0} \in \mathcal{B}^{[-1]}$, since there are $n q-q+n$ terms in the sum, of which one will be zero and the others will be 1 . For all other elements of $G F\left(q^{2}\right)$ it will be zero, since every term in the sum will be 1 . Now $\hat{\sigma}_{q+1}$ takes the same values, and both are of degree $q^{2}-1$. Hence it follows that they are the same, i.e. $\hat{\sigma}_{q+1}=-\sum_{b \in \mathcal{B}}(1-b x)^{q^{2}-1}$. So we get the important relation

$$
z B^{\prime}=\hat{\sigma}_{q+1} B .
$$

Differentiating this, multiplying by $B$ and noting that $B \hat{\sigma}_{q+1}=0(\bmod z)$, we get another useful relation:

$$
B B^{\prime}=B^{2} \hat{\sigma}_{q+1}^{\prime} \quad(\bmod z) .
$$

Differentiating $F(t, x)$ with respect to $x$, we get

$$
F_{x}(t, x)=\left(\sum_{b \in \mathcal{B}} \frac{-b(1-b x)^{q-2} t}{1-(1-b x)^{q-1} t}\right) F(t, x)=\sum_{k=0}^{|\mathcal{B}|}(-1)^{k} \hat{\sigma}_{k}^{\prime} t^{k} .
$$

The terms in the denominator are of the form $\left(1-(1-b x)^{q-1} t\right)$, and for all $x=$ $x_{0} \in G F\left(q^{2}\right)$ this is a factor of $\left(1-t^{q+1}\right)$. Expanding the term in the bracket as a formal power series in $t$, multiplying by $\left(1-t^{q+1}\right)$ and reducing $\bmod z$, we obtain a polynomial $R(t, x)$ of degree at most $q+1$ in $t$ such that

$$
F R=\left(1-t^{q+1}\right) F_{x} \quad(\bmod z)
$$

Comparing coefficients of powers of $t$ we can calculate that the polynomial $R(t, x)$ is of the form

$$
R(t, x)=-\hat{\sigma}_{n}^{\prime}(x) t^{n}+\widehat{R}(t, x) t^{2 n}+\hat{\sigma}_{q+1}^{\prime} t^{q+1},
$$

where $\widehat{R}(t, x)$ is a polynomial containing only powers of $t$ with exponents divisible by $n$. Multiplying the equation by $B$ gives

$$
\left(\sum_{i=0}^{q-q / n+1}(-1)^{i} B \hat{\sigma}_{i n} t^{i n}\right) R=\left(1-t^{q+1}\right) B F_{x} \quad(\bmod z) .
$$

By equating the coefficient of $t^{q+1+n}$ we see that

$$
-\hat{\sigma}_{q+1}^{\prime} B \hat{\sigma}_{n}=-\hat{\sigma}_{q+1+n}^{\prime} B+B \hat{\sigma}_{n}^{\prime} .
$$

Note that since $B \mid \hat{\sigma}_{n}$ we can use the relation $B^{2} \hat{\sigma}_{q+1}^{\prime}=B B^{\prime}(\bmod z)$, and rearranging terms gives

$$
B \hat{\sigma}_{q+1+n}^{\prime}=\left(B \hat{\sigma}_{n}\right)^{\prime} \quad(\bmod z)
$$

Equating successively the coefficient of $t^{i(q+1)+n}$ for $1<i<(n-1)$ gives

$$
B \hat{\sigma}_{i(q+1)+n}^{\prime}=B \hat{\sigma}_{(i-1)(q+1)+n}^{\prime}=\left(B \hat{\sigma}_{n}\right)^{\prime} \quad(\bmod z) .
$$


Since $|B|=n q-q+n$, it follows that $\hat{\sigma}_{(n-1)(q+1)+n} \equiv 0$, and so when we look at the coefficient of $t^{(n-1)(q+1)+n}$ we find that

$$
\left(B \hat{\sigma}_{n}\right)^{\prime} \equiv 0 \quad(\bmod z) .
$$

Since $B \hat{\sigma}_{n}$ has degree at most $(n q-q+n)+n(q-1)<q^{2}$, it follows that $\left(B \hat{\sigma}_{n}\right)^{\prime}=0$ identically, and hence $B \hat{\sigma}_{n}$ is a $p$-th power. Since $B$ does not have multiple factors, this implies that $B^{p-1} \mid \hat{\sigma}_{n}$, which gives a contradiction for $p \neq 2$, since the degree of $\hat{\sigma}_{n}$ is at most $n(q-1)$ and it is not identically zero.

\section{REFERENCES}

[1] S. Ball, A. Blokhuis and F. Mazzocca, Maximal arcs in Desarguesian planes of odd order do not exist, Combinatorica 17, (1997), 31-41. CMP 97:17

[2] A. Cossu, Su alcune proprietà dei $\{k ; n\}$-archi di un piano proiettivo sopra un corpo finito, Rend. Mat. e Appl., 20, (1961), 271-277. MR 25:3419

[3] R. H. F. Denniston, Some maximal arcs in finite projective planes, J. Combin. Theory, 6, (1969), 317-319. MR 39:1345

[4] L. RÉDEI, 'Lückenhafte Polynome über endlichen Körpern, Birkhäuser Verlag, Basel (1970) (English translation: Lacunary polynomials over finite fields, North Holland, Amsterdam, 1973). MR 45:3366; MR 50:4548

[5] J. A. Thas, Construction of maximal arcs and partial geometries, Geom. Dedicata, 3, (1974), 61-64. MR 50:1931

[6] J. A. Thas, Some results concerning $\{(q+1)(n-1) ; n\}-\operatorname{arcs}$ and $\{(q+1)(n-1)+1 ; n\}-\operatorname{arcs}$ in finite projective planes of order $q$, J. Combin. Theory Ser. A, 19, (1975), 228-232. MR 51:13851

[7] J. A. Thas, Construction of maximal arcs and dual ovals in translation planes, Europ. J. Combinatorics, 1, (1980), 189-192. MR 82a:05031

Department of Mathematics, Vrije Universiteit Amsterdam, De Boelelaan 1105, 1081 HV Amsterdam, The Netherlands 